\title{
The prevalence of periarticular lesions detected on magnetic resonance imaging in middle-aged and elderly persons: a cross-sectional study
}

In Je Kim', Dong Hyun Kim², Yeoung Wook Song ${ }^{3}$, Ali Guermazi ${ }^{4}$, Michel D. Crema ${ }^{4}$, David J. Hunter ${ }^{5}$, Young- $\| \mathrm{SeO}^{6}$ and Hyun Ah Kim ${ }^{6,7,8^{*}}$

\begin{abstract}
Background: Previous studies showed that among persons with radiographic knee OA, periarticular lesions were significantly more common among participants with knee pain than those without. However, data were derived mostly from persons with knee $O A$, and there were few normal participants without knee OA in the data analyses. The objectives of this study were to investigate the prevalence of periarticular lesions detected by magnetic resonance imaging (MRI), and to examine their prevalence according to the presence of knee pain and radiographic knee osteoarthritis $(\mathrm{OA})$ in community residents in Korea.
\end{abstract}

Methods: Demographic and knee pain data were obtained by questionnaire from 358 participants of the population-based Hallym Aging Study who were recruited irrespective of the presence of knee OA or pain. Radiographic evaluations consisted of weight-bearing knee anteroposterior radiographs and 1.5-T MRI scans. Periarticular lesions included prepatellar or anserine bursitis, Baker's cyst, and tibiofibular cyst. The prevalence of each lesion in subjects with knee OA or knee pain compared to those without was examined by a chi-square test.

Results: The mean age of the subjects was 72 years and $50.6 \%$ were female. Radiographic knee OA was present in $34.5 \%$. The most prevalent peri-articular lesion was Baker's cyst (27.9\%), followed by tibiofibular cyst (9.5\%). Anserine bursitis and tibulofibular cyst were more common in subjects with knee OA (17.5\% vs $2.2 \%$ for anserine bursitis, $15.8 \%$ vs $6.1 \%$ for tibiofibular cyst in subjects with and without OA, respectively), while Baker's cyst and anserine bursitis were more common in subjects with knee pain (36.3\% vs $21.8 \%$ for Baker's cyst, $14.4 \%$ vs $2.5 \%$ for anserine bursitis in subjects with and without knee pain, respectively).

Conclusions: Periarticular lesions on MRI of the knee are common in middle-aged and elderly persons. Anserine bursitis and Baker's cysts are more common in subjects with knee pain compared to those without.

Keywords: Baker's cyst, Anserine bursitis, Knee pain, MRI, Osteoarthritis, Periarticular lesions

\footnotetext{
* Correspondence: kimha@hallym.ac.kr

${ }^{6}$ Department of Internal Medicine, Hallym University Sacred Heart Hospital, Anyang, Republic of Korea

${ }^{7}$ Institute for Skeletal Aging, Chuncheon, Republic of Korea

Full list of author information is available at the end of the article
} 


\section{Background}

Knee pain is a common musculoskeletal problem in older adults, and is closely related to radiographic osteoarthritis (OA) [1]. However, a substantial number of patients with knee pain do not exhibit radiographic OA [2]. Because recent studies suggest that knee pain is a better predictor of disability than radiographic changes, the precise determination of the causes of knee pain would be important for guiding treatment decisions [3].

Magnetic resonance imaging (MRI) allows clinicians and researchers to evaluate a range of periarticular structures not visualized by conventional radiographs, and MRI can be used for guiding therapeutic decisions. Previous studies showed that among persons with radiographic knee $\mathrm{OA}$, periarticular lesions, such as anserine bursitis and iliotibial band syndrome, were significantly more common among participants with knee pain than those without and may contribute to the pain in these individuals [4]. However, the prevalence of other periarticular lesions, such as prepatellar bursitis, are apparently similar in asymptomatic and symptomatic knee OA [4], suggesting that not all inflammatory lesions in periarticular structures lead to symptoms.

In previous reports on the relationship between periarticular lesions and knee pain, data were derived mostly from persons with knee OA, and there were few normal participants without knee OA in the data analyses [4, 5]. The research questions of this cross-sectional study were: 1) what is the prevalence of periarticular lesions in the community residents according to the presence or absence of knee OA? and 2) what is the prevalence of periarticular lesions in the community residents according to the presence or absence of knee pain?

\section{Methods}

\section{Subjects}

The Hallym Aging Study (HAS) is a prospective cohort study investigating the health of elderly community residents in Chuncheon, a city $\sim 120 \mathrm{~km}$ east of Seoul, Korea. This ongoing study began in 2004, with follow-up examinations scheduled every 3 years. Its methods have been described in detail elsewhere [6]. Briefly, 1489 subjects among 71,061 residents aged $\geq 50$ years in Chuncheon were selected randomly and contacted. Participants were invited both by the telephone and mail to the study with the announcement that "This is a study evaluating general health and physical function in the elderly." Finally 568 subjects refused to participate or were ineligible and 918 subjects finished the first wave in year 2004. Data on OA were collected from the $2^{\text {nd }}$ wave, which began in year 2007. Among the first wave subjects, 90 refused to participate, 13 could not be contacted, 64 had died, and 49 had moved from the area, and 702 subjects were eligible. Of them, 129 refused to undergo the OA substudy; they were significantly older than those who participated (72.3 vs. 70.4 years, $p<0.01$ ), although the sex ratio was not different. Of the 573 remaining subjects, 400 were chosen randomly for knee MRIs regardless of the presence of knee pain or of radiographic knee OA. Of them, 36 declined to undergo knee MRI, three had previously undergone bilateral total knee replacements, and three had other contraindications to MRI, leaving 358 subjects who underwent knee MRIs. The Ethics Committee of Hallym University approved the study protocol. Written informed consent was obtained from all study participants.

\section{Data collection}

Demographic information was collected using a standard questionnaire. Knee pain was assessed by asking, "Did you have pain, aching, or stiffness in either of your knees in the last month?" Those who answered "yes" was considered to have knee pain. All participants were also evaluated using the Western Ontario and McMaster Universities (WOMAC) Osteoarthritis Index for evaluating pain and self-reported functional status [7]. Height $(\mathrm{cm})$ and body weight $(\mathrm{kg})$ were measured to the nearest $0.1 \mathrm{~cm}$ and $0.1 \mathrm{~kg}$, respectively, with subjects wearing light clothing and barefoot, and body mass index (BMI) was calculated as the weight in $\mathrm{kg}$ divided by the square of the body height in meter.

\section{Radiographic assessment}

Radiographic evaluations consisted of $14 \times 17$-in., anteroposterior radiographs taken during weight bearing with a semi-flexed knee. A Plexiglas frame (SynaFlexor, San Francisco, CA, USA) was used to standardize knee position according to the manufacturer's recommendations. Each knee was graded using the KellgrenLawrence (K-L) grading scale [8]. Radiographic knee OA was defined as present if the participant had a radiographic $\mathrm{K}-\mathrm{L}$ grade $\geq 2$ in the tibiofemoral joint. All radiographs were read twice at an interval of at least 2 weeks by one reader, an academically based rheumatologist (HAK) who was unaware of the knee pain status or MRI findings. The intra-reader reproducibility of assessments was high, with weighted kappa values of 0.86 (95\% confidence interval, $\mathrm{CI}=0.80$ 0.91 ) for the $\mathrm{K}-\mathrm{L}$ grade and 0.89 (95\% CI =0.83-0.94) for $\mathrm{K}-\mathrm{L}$ grades $0-1$ (non-OA) versus $\mathrm{K}-\mathrm{L}$ grade $\geq 2$ (OA). Images that were assigned different $\mathrm{K}-\mathrm{L}$ grades at the two readings were adjudicated by consensus between the original reader and a second reader (DJH). The weighted kappa value for inter-reader reliability was 0.84 for non-OA versus OA (95 \% CI =0.67-1.01). 


\section{MRI and evaluation of periarticular lesions}

Each participant underwent an MRI of one knee. If both knees were symptomatic, the more symptomatic knee was chosen. For subjects without knee pain, the dominant knee was selected for imaging. Knee dominance was determined by the following question: "When you start to kick a ball, which foot would you use first?" MRI scans of the knee were obtained using a 1.5-T scanner (Philips, Andover, MA, USA) with a phase-array knee coil.

The imaging protocol included a sagittal 3D waterselect excited cartilage three-fluid sequence (repetition time $20 \mathrm{~ms}$, echo time $7.8 \mathrm{~ms}$, slice thickness $3.0 \mathrm{~mm}$, interslice gap $1.5 \mathrm{~mm}$, field of view $150 \times 150 \mathrm{~mm}$, and matrix, $304 \times 304$ ), a sagittal T2-weighted fat-suppression sequence (repetition time $2789.6 \mathrm{~ms}$, echo time $60 \mathrm{~ms}$, slice thickness $4.0 \mathrm{~mm}$, interslice gap $0.4 \mathrm{~mm}$, echo spacing $13.3 \mathrm{~ms}$, field of view, $160 \times 160 \mathrm{~mm}$, and matrix $208 \times 166)$, sagittal proton density-weighted fat-suppression sequence (repetition time $2000 \mathrm{~ms}$, echo time $15 \mathrm{~ms}$, slice thickness $4.0 \mathrm{~mm}$, interslice gap $0.4 \mathrm{~mm}$, echo spacing $15 \mathrm{~ms}$, field of view $160 \times$ $160 \mathrm{~mm}$, and matrix, $224 \times 224$ ), coronal water-select excited cartilage three-fluid sequence (repetition time $20 \mathrm{~ms}$, echo time $7.7 \mathrm{~ms}$, slice thickness $3 \mathrm{~mm}$, interslice gap $1.5 \mathrm{~mm}$, field of view $160 \times 160 \mathrm{~mm}$, and matrix $304 \times 304)$, and axial T2-weighted fast-field echo sequence (repetition time $439.2 \mathrm{~ms}$, echo time $13.8 \mathrm{~ms}$, slice thickness $4 \mathrm{~mm}$, interslice gap $0.4 \mathrm{~mm}$, field of view $140 \times 140 \mathrm{~mm}$, and matrix $256 \times 256$ ).

The MRI scans were read by an experienced musculoskeletal radiologist (MDC) who was unaware of participants' characteristics as well as clinical and radiographic data. Semi-quantitative assessments of the periarticular lesions were performed using the Whole-Organ MRI Score (WORMS) [9]. Baker's cyst was scored from 0 to $3(0=$ none, $1=$ small, $2=$ medium, and $3=$ large $)$. Anserine and prepatellar bursitis, and tibiofibular cysts were scored as 0 or $1(0=$ none, $1=$ present $)$. A random subset of images $(n=38)$ was re-read to determine intra-observer reproducibility $(\kappa=1.00(1.00-1.00)$ for anserine bursitis, $0.64(0.15-1.00)$ for prepatellar bursitis, 0.91 (0.72-1.00) for Baker's cyst, and 1.00 (1.001.00) for tibiofibular cyst).

\section{Statistical analysis}

We calculated the prevalence of periarticular lesions according to the presence of radiographic knee OA or knee pain. The prevalence of periarticular lesions in subjects with knee OA or knee pain was compared to those without using a chi-square test. All tests were two-tailed, and $P$-values $<0.05$ were considered to indicate statistical significance. Analyses were performed using the SAS software (ver. 9.1; SAS Institute, Cary, NC, USA).

\section{Results}

Among the 358 participants, 10 patients were excluded in the data analysis because only unilateral knee radiographs were taken due to a clerical error. Among 348 participants, the mean age was 72 years, and $50.6 \%$ were female (Table 1). Radiographic OA and pain in the scanned knee were present in $34.5 \%$ (14.5\% in males, $54.0 \%$ in females) and $42.0 \%$ (25.6 \% in males, $58.0 \%$ in females), respectively.

Periarticular lesions were present in $45.4 \%$ of all participants (Table 2). The most prevalent lesion was a Baker's cyst (27.9\%), followed by a tibiofibular cyst $(9.5 \%)$ and anserine bursitis $(7.5 \%)$. Periarticular lesions were present in 66.7 and $33.3 \%$ of participants with and without knee OA, respectively. Anserine bursitis and tibiofibular cyst were significantly more common in the participants with knee OA than in those without it. Women had a higher prevalence of anserine bursitis $(12.5 \%$ in women, $1.7 \%$ in men, $p<0.0001)$ and tibiofibular cysts than men (14.2\% in women, $4.1 \%$ in men, $p=0.0001$ ) (data not shown).

Next, the prevalence of periarticular lesions according to the presence of knee pain was examined. (Table 3). Periarticular lesions were present in 60.1 and $33.5 \%$ of participants with and without knee pain, respectively. Baker's cyst and anserine bursitis were present in 34.6 and $14.4 \%$ of participants with knee pain, respectively, which was significantly more prevalent compared to those without.

\section{Discussion}

Periarticular lesions were common, affecting $45 \%$ of these community-based older adults. The most prevalent lesion was Baker's cyst (27.9 \%), followed by tibiofibular cyst and anserine bursitis. Periarticular lesions were more prevalent in subjects with OA than in those without OA. Anserine bursitis and tibiofibular cyst were more common in subjects with knee OA, while Baker's cyst and anserine bursitis were more common in subjects with knee pain.

Benign cystic lesions are frequently seen on MRI of the knee. Baker's cysts, the most frequently encountered,

Table 1 Demographic and clinical characteristics of the subjects

\begin{tabular}{ll}
\hline Age, mean \pm SD years & Subjects $(n=348)$ \\
Female & $71.7 \pm 5.8$ \\
BMl, mean \pm SD kg/m & $176(50.6)$ \\
Manual occupation & $27.3 \pm 52.4$ \\
Radiographic knee OA & $72(20.7)$ \\
Knee pain in the last month & $120(34.5)$ \\
\hline
\end{tabular}

Values are number (percentage) of subjects, unless otherwise indicated $S D$ standard deviation, $B M I$ body mass index, $O A$ osteoarthritis 
Table 2 Prevalence of periarticular lesions according to the presence of radiographic knee $O A$

\begin{tabular}{llll}
\hline & OA $(n=120)$ & Non-OA $(n=228)$ & $P$-value \\
\hline Baker's cyst & $39(32.5)$ & $58(25.4)$ & 0.163 \\
Tibiofibular cyst & $19(15.8)$ & $14(6.1)$ & 0.003 \\
Anserine bursitis & $21(17.5)$ & $5(2.2)$ & $<0.001$ \\
Prepatellar bursitis & $2(1.7)$ & $0(0.0)$ & 0.118 \\
\hline
\end{tabular}

Data presented are number of lesions (percentage)

$O A$ osteoarthritis

is not a true cyst, but a fluid collection in the semimembranosus-medial gastrocnemius bursa. MRI studies have shown that the prevalence of Baker's cysts is as high as $40 \%[4,10,11]$. There is conflicting data regarding the association of Baker's cysts with radiographic knee OA. Although a higher prevalence of Baker's cyst was reported in those with than without knee OA [10, 12], others reported no difference in its prevalence, consistent with our result [13]. In people with frequent chronic pain, no trend was observed between its presence and increasing radiographic knee OA severity [11].

Tibiofibular cysts, which are caused by an increase in intra-articular pressure due to active synovitis or joint injury, cause an outpouching of the tibiofibular joint capsule, herniating to form the synovial cyst [11]. Although we found that tibiofibular cysts were more common in subjects with knee OA, previous results did not reveal significant association between tibiofibular cysts and knee OA after adjustment of confounders [11, 14].

Chronic anserine bursitis, a common lesion in elderly patients who have OA or rheumatoid arthritis [15], was reported not to be significantly associated with knee OA in two recent case-control studies $[14,16]$. On the other hand, our study showed higher prevalence of anserine bursitis in knee OA subjects, and the discrepancy may be due to differences in the populations studied or the detection limits. Also, because only a small number of lesions were available for analysis, further longitudinal studies with a larger sample size will be helpful in this regard.

In this study, women had a higher prevalence of anserine bursitis and tibiofibular cysts than men. These results may be in part related to a higher prevalence of knee OA among women and other multifactorial factors.
Because cartilage is avascular and aneural, the pain in $\mathrm{OA}$ is not likely to result from cartilage damage per se, and may arise from structures outside the joint cavity $[2,17,18]$. We found that Baker's cyst and anserine bursitis were more prevalent in subjects with knee pain. In previous studies, Baker's cyst was also associated with weight-bearing pain and painful flare in OA patients $[5,19]$. However, other reports have shown no relationship between Baker's cyst, anserine bursitis, and pain in people with OA $[10,14,20]$. The presence of Baker's cyst in knees with osteoarthritic pain has been shown to correlate with synovial inflammation [12]. Inflammation from various local and systemic processes, such as overuse, trauma, infection, hemorrhage, internal joint derangement, and inflammatory arthropathy, leads to thickening of the synovial lining and fluid accumulation within the bursae around the knee joint [15]. Acute anserine bursitis results frequently from overuse injury or trauma, especially in runners, causing medial knee pain, whereas chronic anserine bursitis occurs in patients with degenerative joint disease or rheumatoid arthritis [15, 16, 21]. Knee pain arising from it may be determined by multiple sources, including not only structural damage, pain processing mechanism, and gender, but also activities of daily living provoking the pain, which may depend on cultural differences among the populations studied.

This study has several limitations. First, only anterioposterior knee radiographs were obtained, preventing the evaluation of patellofemoral OA. Second, because we did not have surgical or pathological confirmation, some of the lesions, such as bursitis, may have been a simple collection of fluid without inflammation. Third, periarticular lesions other than the ones included were found infrequently, so that we could not subject them to statistical analyses. Finally, although we examined cartilage morphology including signal change and thickness, we did not measure cartilage volume in our study, which could be a limitation in the data analysis.

\section{Conclusions}

This study is the first reported population-based research using MRI to investigate the relationships between knee pain and periarticular lesions in Asian subjects. Because

Table 3 Prevalence of periarticular lesions according to the presence of knee pain in the last month

\begin{tabular}{llll}
\hline & Knee pain $(n=146)$ & No knee pain $(n=202)$ & $P$-value \\
\hline Baker's cyst & $53(36.3)$ & $44(21.8)$ & 0.003 \\
Tibiofibular cyst & $16(11.0)$ & $17(8.4)$ & 0.697 \\
Anserine bursitis & $21(14.4)$ & $5(2.5)$ & $<0.001$ \\
Prepatellar bursitis & $2(1.4)$ & $0(0.0)$ & 0.244 \\
\hline
\end{tabular}

Data presented are number of lesions (percentage)

$O A$ osteoarthritis 
baker's cyst and anserine bursitis were significantly more common in subjects with knee pain, specific treatment of this lesion in addition to systemic analgesic treatment might further improve pain in knee OA patients.

\section{Availability of data and materials}

Data will not be shared because of the policy of the Hallym Aging Study principal investigator (DH Kim).

\section{Abbreviations}

BMI: body mass index; K-L: Kellgren-Lawrence; MRI: magnetic resonance imaging; OA: osteoarthritis; WOMAC: Western Ontario and McMaster Universities; WORMS: Whole-Organ MRI Score.

\section{Competing interests}

Ali Guermazi is president of Boston Imaging Core Lab (BICL), and consultant to Genzyme, Astra Zeneca, Merck Serono and Pfizer. Michel Crema is vice president of BICL. All other authors have declared no conflicts of interest.

\begin{abstract}
Authors' contributions
IK performed the statistical analysis and wrote the manuscript. DHK participated in provision of study materials or patients, and technical or logistic support. YWS performed analysis of the data, and obtained funding. AG, MDC, and DJH participated in interpretation, collection, and assembly of the data. YIS conceived of the study. HAK conceived of the study, supervised data analysis, wrote the manuscript, and obtained funding. All authors interpreted the data, and revised the manuscript critically for intellectual content, and read and approved the final manuscript.
\end{abstract}

\section{Funding statement}

This study was supported by a grant from the Korea Health Technology R\&D Project, Ministry of Health \& Welfare, Republic of Korea (A1 10274, HI14C2248). The study sponsor was not involved in the study design, data collection, analysis and interpretation of data; in the writing of the manuscript; and in the decision to submit the manuscript for publication.

\section{Author details}

${ }^{1}$ Department of Internal Medicine, Ewha Womans University Mokdong Hospital, Seoul, Republic of Korea. ${ }^{2}$ Department of Social and Preventive Medicine, Hallym Research Institute of Clinical Epidemiology, Hallym University, Chuncheon, Republic of Korea. ${ }^{3}$ Department of Internal Medicine, Seoul National University Hospital, Seoul, Republic of Korea. ${ }^{4}$ Department of Radiology, Boston University School of Medicine, Boston, MA, USA. ${ }^{5}$ Department of Rheumatology, Royal North Shore Hospital and Institute of Bone and Joint Research, Kolling Institute, University of Sydney, Sydney, Australia. ${ }^{6}$ Department of Internal Medicine, Hallym University Sacred Heart Hospital, Anyang, Republic of Korea. 'Institute for Skeletal Aging, Chuncheon, Republic of Korea. ${ }^{8}$ Division of Rheumatology, Department of Internal Medicine, Hallym University Sacred Heart Hospital, 896, Pyongchondong, Dongan-gu, Anyang, Kyunggi-do 431-070, Republic of Korea.

Received: 6 November 2015 Accepted: 15 April 2016

Published online: 26 April 2016

\section{References}

1. Urwin M, Symmons D, Allison T, Brammah T, Busby H, Roxby M, et al. Estimating the burden of musculoskeletal disorders in the community: the comparative prevalence of symptoms at different anatomical sites, and the relation to social deprivation. Ann Rheum Dis. 1998;57:649-55.

2. Bedson J, Croft PR. The discordance between clinical and radiographic knee osteoarthritis: a systematic search and summary of the literature. BMC Musculoskelet Disord. 2008;9:116.

3. Hadler NM. Knee pain is the malady - not osteoarthritis. Ann Intern Med. 1992:116:598-9.

4. Hill CL, Gale DR, Chaisson CE, Skinner K, Kazis L, Gale ME, et al. Periarticular lesions detected on magnetic resonance imaging: prevalence in knees with and without symptoms. Arthritis Rheum. 2003;48:2836-44.

5. Cao Y, Jones G, Han W, Antony B, Wang X, Cicuttini F, et al. Popliteal cysts and subgastrocnemius bursitis are associated with knee symptoms and structural abnormalities in older adults: a cross-sectional study. Arthritis Res Ther. 2014:16:R59.

6. Kim I, Kim HA, Seo Yl, Song YW, Jeong JY, Kim DH. The prevalence of knee osteoarthritis in elderly community residents in Korea. J Korean Med Sci. 2010;25:293-8.

7. Bellamy N, Buchanan WW, Goldsmith CH, Campbell J, Stitt LW. Validation study of WOMAC: a health status instrument for measuring clinically important patient relevant outcomes to antirheumatic drug therapy in patients with osteoarthritis of the hip or knee. J Rheumatol. 1988:15:1833-40.

8. Kellgren JH, Lawrence JS. Radiological assessment of osteoarthrosis. Ann Rheum Dis. 1957:16:494-502

9. Peterfy CG, Guermazi A, Zaim S, Tirman PF, Miaux Y, White D, et al. Wholeorgan magnetic resonance imaging score (WORMS) of the knee in osteoarthritis. Osteoarthr Cartil. 2004;12:177-90.

10. Hill CL, Gale DG, Chaisson CE, Skinner K, Kazis L, Gale ME, et al. Knee effusions, popliteal cysts, and synovial thickening: association with knee pain in osteoarthritis. J Rheumatol. 2001;28:1330-7.

11. Hayashi D, Roemer FW, Dhina Z, Kwoh CK, Hannon MJ, Moore C, et al. Longitudinal assessment of cyst-like lesions of the knee and their relation to radiographic osteoarthritis and $\mathrm{MRI}$-detected effusion and synovitis in patients with knee pain. Arthritis Res Ther. 2010;12:R172.

12. Chatzopoulos D, Moralidis E, Markou P, Makris V, Arsos G. Baker's cysts in knee with chronic osteoarthritic pain: a clinical, ultrasonographic, radiographic and scintigraphic evaluation. Rheumatol Int 2008:29:141-6.

13. Tschirch FT, Schmid MR, Pfirrmann CW, Romero J, Hodler J, Zanetti M. Prevalence and size of meniscal cysts, ganglionic cysts, synovial cysts of the popliteal space, fluid-filled bursae, and other fluid collections in asymptomatic knees on MR imaging. AJR Am J Roentgenol. 2003;180:1431-6.

14. Guermazi A, Hayashi D, Roemer FW, Niu J, Yang M, Lynch JA, et al. Cyst-like lesions of the knee joint and their relation to incident knee pain and development of radiographic osteoarthritis: the MOST study. Osteoarthr Cartil. 2010;18:1386-92.

15. McCarthy $C L$, McNally EG. The MRI appearance of cystic lesions around the knee. Skeletal Radiol. 2004;33:187-209.

16. Alvarez-Nemegyei J. Risk factors for pes anserinus tendinitis/bursitis syndrome: a case control study. J Clin Rheumatol. 2007;13:63-5

17. Fortier LA, Nixon AJ. Distributional changes in substance $P$ nociceptive fiber patterns in naturally osteoarthritic articulations. J Rheumatol. 1997;24:524-30.

18. Muraki S, Oka H, Akune T, Mabuchi A, En-yo Y, Yoshida M, et al. Prevalence of radiographic knee osteoarthritis and its association with kneepain in the elderly of Japanese population-based cohorts: the ROAD study. Osteoarthr Cartil. 2009:17:1137-43

19. de Miguel ME, Cobo Ibanez T, Uson Jaeger J, Bonilla Hernán G, Martín ME. Clinical and ultrasonographic findings related to knee pain in osteoarthritis. Osteoarthr Cartil. 2006;14:540-4.

20. Kornaat PR, Bloem JL, Ceulemans RYT, Riyazi N, Rosendaal FR, Nelissen RG, et al. Osteoarthritis of the knee: association between clinical features and MR imaging findings. Radiology. 2006:239:811-7.

21. Janzen DL, Peterfy CG, Forbes JR, Tirman PFJ, Genant HK. Cystic lesions around the knee joint: MR imaging findings. AJR Am J Roentgenol. 1994; 163:155-61.
Submit your next manuscript to BioMed Central and we will help you at every step:

- We accept pre-submission inquiries

- Our selector tool helps you to find the most relevant journal

- We provide round the clock customer support

- Convenient online submission

- Thorough peer review

- Inclusion in PubMed and all major indexing services

- Maximum visibility for your research

Submit your manuscript at www.biomedcentral.com/submit 